\title{
Diabetic Maculopathy Detection using Image Processing
}

\author{
J. Pradeepkandhasamy, S. Balamurali, M. Arun, M. Mariappan
}

\begin{abstract}
Diabetic Retinopathy (DR) is a serious eye disease caused to human beings having diabetics. DR will affect the retina of the eye and even it may lead to complete blindness. It is essential to have an early treatment for the diagnosis of $D R$ to avoid blindness. There are many physical tests like visual test, pupil dilation to detect retinopathy but all are time consuming processes. For diabetic retinopathy, it needs a continuous monitoring process. The main objective of this work is to detect diabetic maculopathy which is one of the major retinal abnormalities found among diabetic persons. Diabetic maculopathy is detected using image processing technique. In image processing techniques, we use image pre processing to reduce the noise and use segmentation process to extract the features of the macula. After that the features are compared using the classifier algorithm and the performances are measured using the accuracy, sensitivity and specificity.
\end{abstract}

Keywords : Classification , Retinopathy, Diabetic maculopathy Image processing, Segmentation

\section{INTRODUCTION}

Now a days diabetic is one of the major and dangerous diseases by which the entire human community is suffering. Currently all age group people get for diabetic. The person with diabetic not undergoing a regular treatment and monitoring process gets in to diabetic retinopathy. Diabetic Retinopathy is a medical abnormality caused to human eye which affects the retina due to the leakage of fluid from blood vessels to retina. The fluid from the blood vessels affects the sensitive tissues at the retina. Diabetic Retinopathy (DR) is classified in to two major types; one is proliferative diabetic retinopathy (PDR) and other is non-proliferative diabetic retinopathy (NPDR). In NPDR the disease severity can be categorized in to mild, moderate and sever. In manual treatment monitoring and detecting DR is a time consuming process. To avoid that computer aided systems can be built to reduce the manual process and make the system fast and more accurate in detecting the severity of the disease. The main aim of computer aided systems is to give guidance to the ophthalmologist for the prediction of diabetic maculopathy.
The main drawback of the computer aided systems is the accuracy of the prediction. In this work, we develop a computer aided system which detects diabetic maculopathy in an effective manner using image processing techniques. The system is built using MATLAB under windows environment. MATLAB is chosen due to its powerful support towards data analysis and visualization.

\section{LITERATURE SURVEY}

In [2] the authors implemented a method to detect the optic nerve in fundus images and they used a novel algorithm called fuzzy convergence to detect the origin of blood vessel network. Their algorithm has been compared with some other similar algorithms like least squares and Hough space based and found their algorithm performs well and produce $89 \%$ accuracy in correct detection of images.

In [3], different methods of color image segmentation have been discussed by the authors and a comparative study has been done to find out the algorithm for segmentation process.

Optic Disk (OD) detection is the vital step in developing a system which detects the severity of DR. In [4], they developed a system which detects DR which uses a template based method for segmentation OD. In this method, first the OD containing in the sub image is extracted from the main image and then the optic disk pixel and its surrounding regions are selected. From this study, the authors concluded that circular approach for OD segmentation shows high success rate. [5] proposed another adaptive method for automatic segmentation of OD from color fundus images. The approach has been used to detect the vascular tree and then algorithm for locating OD is used after locating the OD the OD boundaries has been detected. The proposed method uses DRIVE and DIARETDB1 databases for experimental verification and conclude that the proposed method has high performance that any other similar methods for OD segmentation and OD boundary detection.

Revised Manuscript Received on December 16, 2019.

* Correspondence Author

J. Pradeepkandhasamy*, Department of Computer Applications, School of Computing, Kalasalingam Academy of Research and Education, India. Email: j.pradeepkandasamy@klu.ac.in

S. Balamurali, Department of Computer Applications, School of Computing, Kalasalingam Academy of Research and Education, India. Email: sbmurali@rediffmail.com

M. Arun, Department of Computer Applications, School of Computing, Kalasalingam Academy of Research and Education, India. Email: m.arunvincent@gmail.com

M. Mariappan, Department of Computer Applications, School of Computing, Kalasalingam Academy of Research and Education, India .Email: apexraja98@gmail.com 


\section{PROPOSED METHODOLOGY}

The architecture of the proposed system is given below.

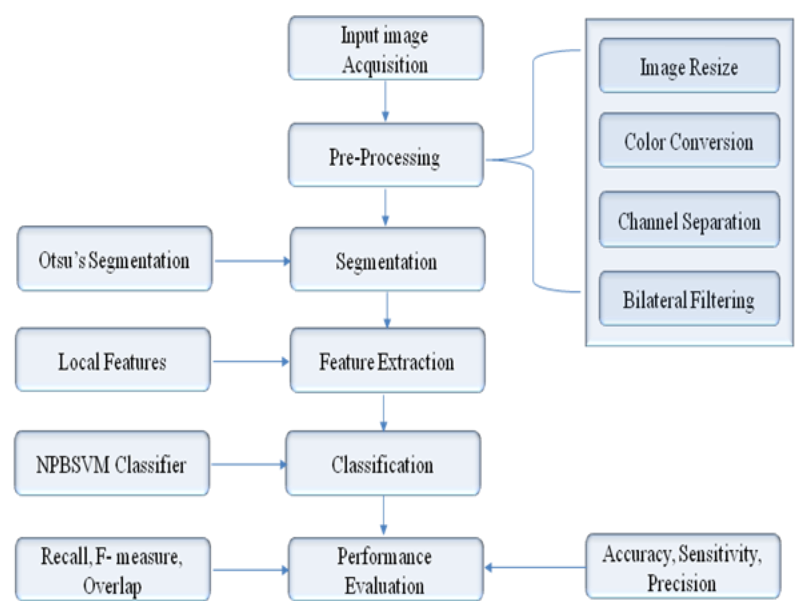

Fig 1. Proposed System Flow

\section{A. Image Acquisition}

This is the first step in most of the computer aided design (CAD) system that is used for the detection of DR. Here we get the input images from the publicly available databases such as DIARETDB1, Indian Diabetic Retinopathy Image Dataset (IDRiD), Structured Analysis of Retina (STARE) database. The images are obtained from the dataset as a query image.

\section{B. Pre Processing}

After getting input of the images, all of them are pre processed using the following techniques:

i. Image Resizing - All the images are resized into $256 \mathrm{X}$ 256 sized images for the processing.

ii. Color conversion - All the color images are converted in to gray scale image so that it's easy to further processing. If more color involves, then our processing task will become difficult.

iii. Channel Separation - An RGB image will contain three channels but our gray scale images will have only one channel. It is easy to separate the grey and white channels.

iv. Bilateral Filtering - Filtering step is done to enhance some features of images. Bilateral filtering is a smoothing filter which will maintain the originality of the edges in images. It is also a noise reducing filter.

\section{Segmentation}

Segmentation is a common technique in digital image processing and analysis. It is the process of dividing the images in to a group of pixels or regions based on their characteristics. It involves the separation of foreground image from the background image. There are many segmentation algorithms available, however in our work we implemented Otsu's segmentation algorithm for segmentation process. Otsu's method performs thresholding on two dimensional and three dimensional gray scale images to create a binary image.
In Otsu's method, the interclass variance of pixels is minimized using a threshold value [6]. Using this, macula region is segmented. The resulted image will be threshold by automatic selected threshold level [7]. The threshold value is the normalized value in the range $[0,1]$. In [8] minimize inter class variance is defined as a weighted sum of variances of the two classes:

$$
\sigma_{\omega}^{2}(t)=\omega_{0}(t) \sigma_{0}^{2}(t)+\omega_{1}(t) \sigma_{1}^{2}(t)
$$

Here weights $\omega_{0,1}$ are the probability of two classes which are separated by threshold $\mathrm{t}, \sigma_{0,1}$ are the variance of two classes. The class probability $\omega_{0,1}$ is then computed

$$
\begin{aligned}
& \omega_{0}(t)=\sum_{i=0}^{t-1} p(i) \\
& \omega_{1}(t)=\sum_{i=t}^{L-1} p(i)
\end{aligned}
$$

In this segmentation process, we can also find the thickness of the blood vessel present in the macula region.

\section{Feature Extraction}

Human eye has many features such as blood vessels, Exudates, Microaneurysums, Optic disk, Edema and local features like thickness, size, Local binary patterns, color Moments etc. are used to identify the severity of the DR [9]. In our work the local features which play important role in identifying the severity of DR is extracted and given as an input for the classification algorithm.

\section{E. Clasification}

Classification is the process of categorization of related objects or pixels. Classification is an image analysis process. Image classification process analysis the properties of the image features and organize the data into categories. Classification stage involves two phases one is training phase and other is testing phase. Several classification algorithms are available in the market among them Support Vector Machine (SVM) algorithm plays a vital role. In our work, we propose a novel Probabilistic Neural Network (PNN) based SVM algorithm for classification. PNN is a feed forward neural network mainly used for classification and pattern recognition. When the input is given the first layer computes the distance between the input vector and training input vector and it will produce a vector whose elements are close to the training input. The second layer sums all these for a class of input to produce net output vector of probabilities. As a final the second layer picks the maximum of these probabilities and produce 1 or 0 for the classes. The architecture of PNN is given in Fig. 2. 


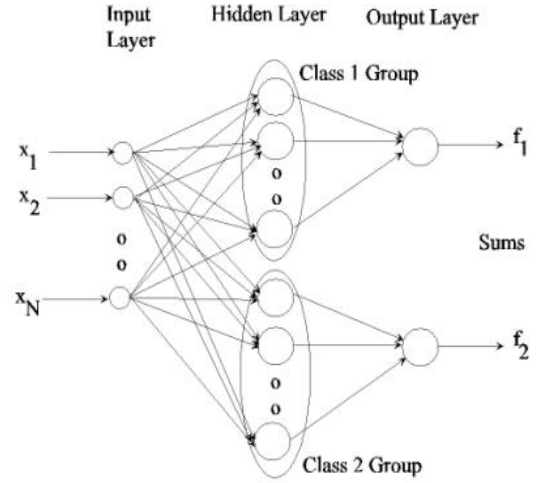

Fig. 2. Architecture of PNN

The following is the high level algorithm which briefs how PNN works.

Step 1: reads input vector and feed to the network layer in each class.

Step 2: for each:

hidden nodes, it computes the Gaussian function value.

Step 3: for each

group of hidden node, assign all the input functional values to the output node.

Step 4: add all the inputs and multiply the sum by a constant at each output node.

Step 5: find the maximum value at each node.

SVM is a supervised learning method for classification [10]. SVM uses training vectors and pair of input-output to build a model. That model is used to predict the class to which the data belong. svmtrain and svmclassify are the two Matlab functions used in classification of data. As a result of this stage, we classify two classes one is a normal macula and other is an abnormal stage of macula candidate in the dataset.

\section{F.Performance Evaluation}

After the classification of candidates in the dataset is over, the performance of our classifier is evaluated based on Accuracy, sensitivity, specificity, F1-Score, Overlap values. The accuracy, sensitivity and specificity values can be classified as in [11].

\section{EXPERIMENTAL RESULTS}

A detailed analysis has been made to study the Performance of our proposed system to detect diabetic maculopathy. Our system was implemented in MATLAB $\mathrm{R} 2015 \mathrm{~b}$ with windows operating system. Correctness of the proposed system is evaluated in terms of accuracy that is how accurately detecting the presence of pathologies. For our experimental evaluation we have taken images from the publicly available retinal dataset DIARETDB1 [12]. Fig. 3 is the input image taken for our work. Then the input image is converted in to gray scale image and subsequently the red, blue and green channel images are also obtained using color conversion technique which is shown in Fig. 4a and Fig. $4 \mathrm{~b}$. The below mentioned figure shows the results obtained in our work.

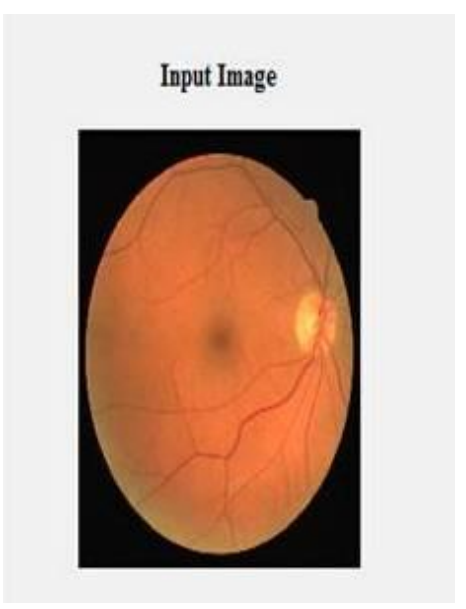

Fig. 3. Input image

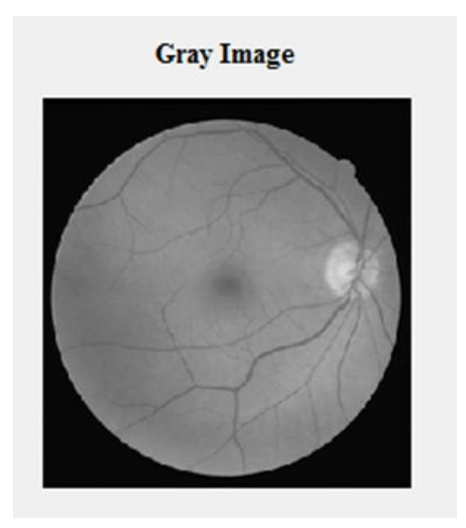

Fig. 4a. Gray Scale Image

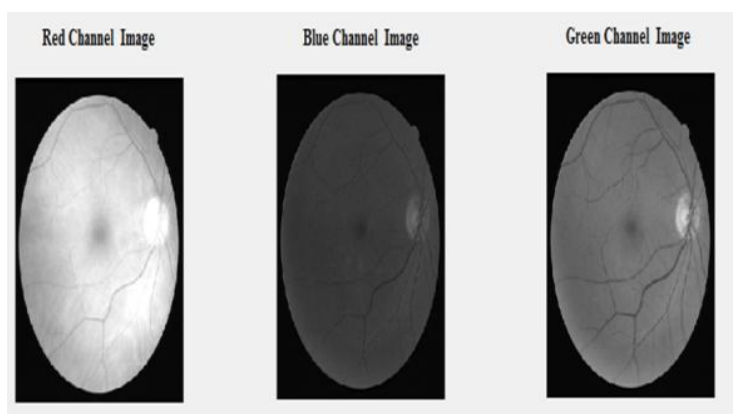

Fig. 4b. Color converted Images

After the channel separation by applying filter, we get the filtered image i.e. enhanced image which is ready for extracting the required features for predicting the severity of DR. Fig 5 shows the blood vessel extracted and macula detected image along with some feature values. 


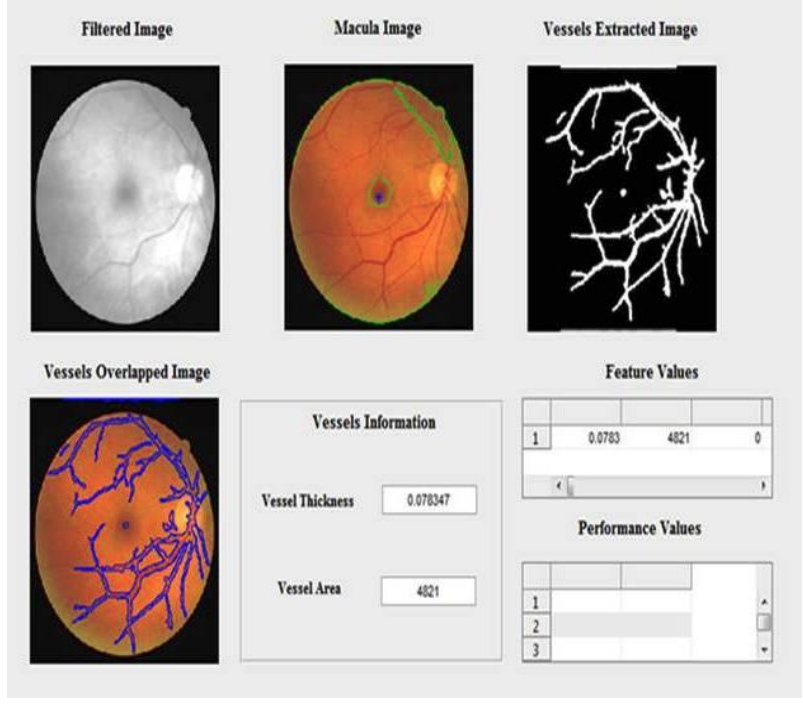

Fig. 5. Feature extracted image and feature values

After the features and feature values are extracted, then the classification is performed to classify the severity of the input image. The input image is classified under three categories normal, mild and sever stage image as shown in Fig. 6

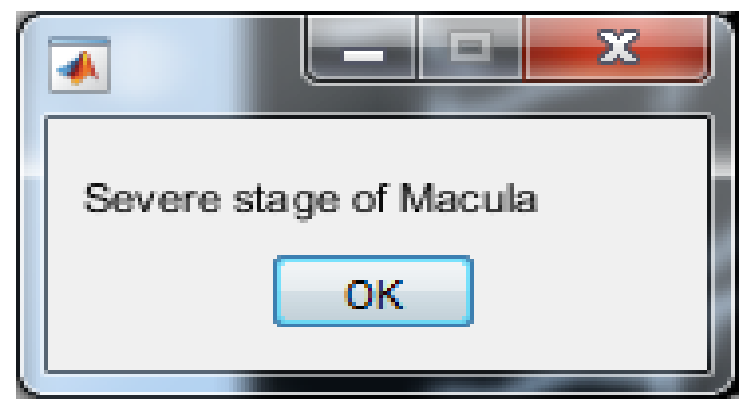

Fig. 6 Classification result

The classification performance is assessed using the performance evaluation criteria like sensitivity, specificity and accuracy values. The specificity and sensitivity values are defined as specified in [11]. In our work, we achieved the accuracy as $92 \%$, specificity as $94 \%$ and sensitivity as $89 \%$ which is better than the most of the state-of-the-art approach. Table I shows the comparison of our work with already existing work

Table I Comparison result of various works

\begin{tabular}{|l|c|c|c|}
\hline \multirow{2}{*}{\multicolumn{1}{c|}{ Author }} & \multicolumn{3}{c|}{ Performance Evaluation } \\
\cline { 2 - 4 } & Sensitivity & Specificity & Accuracy \\
\hline Rocha et al.[13] & - & - & 0.8810 \\
\hline S.S. Rahim et al[14] & 0.859 & 0.65 & 0.7861 \\
\hline Proposed work & 0.89 & 0.94 & 0.92 \\
\hline
\end{tabular}

\section{CONCLUSIONS}

In this study we proposed a novel technique for detecting the maculopathy in diabetic patients using the image processing technique. We designed a system in GUI environment which will assist the ophthalmologist in taking decision regarding the disease severity of patients. There are many algorithms and techniques available to detect diabetic maculopathy our system performs well when compared with other algorithms or techniques used in some other state of art approach.

\section{REFERENCES}

1. D. Sidibe, I. Sadek, and F. Meriaudeau, "Discrimination of retinal images

containing bright lesions using sparse coded features and SVM,' Computers in Biology and Medicine., vol. 62, pp. 175-184, 2015

2. A. Hoover and M. Goldbaum, "Locating the optic nerve in a retinal image using the fuzzy convergence of the blood vessels", IEEE Transactions on Medical Imaging, Vol. 22, No. 8, pp. 951 - 958, 2003

3. N. Sharma, M. Mishra, M. Shrivastava," Colour image segmentation techniques and issues: An approach", International Journal of Scientific \& Technology Research Vol. 1, No 4, pp. 9-12, 2012

4. A. Aquino, M. E. Gegúndez-Arias, and D. Marín,’’ Detecting the optic disc boundary in digital fundus images using morphological, edge detection, and feature extraction techniques, IEEE Transactions on Medical Imaging, Vol. 29, No. 11, pp. 1860 - 1869, November 2010

5. D. Welfera , J. Scharcanski, Cleyson. M. Kitamura, Melissa. M. Dal Pizzol, Laura W. B. Ludwig, D. R. Marinho "Segmentation of the optic disk in color eye fundus images using an adaptive morphological approach", Computers in Biology and Medicine, Vol 40, No 2, pp. 124-137, 2010

6. N. Otsu, "A Threshold selection method from gray level histograms," IEEE Transactions on Systems, Man, and Cybernetics, Segmentation of the optic disk in color eye fundus images using an adaptive morphological approach Vol. 9, No. 1, pp. 62-66, 1979

7. A. Sopharak, B. Uyyanonvara , S. Barman, Thomas H. Williamson, "Automatic detection of diabetic retinopathy exudates from nondilated retinal images using mathematical morphology methods" , Computerized Medical Imaging and Graphics, Vol 32, No 8, pp 720-727, 2008

8. S. Hazra, A. Patra , T. U. Paul "Exudates detection of retinal images using Otsu's thresholding and Kirsch's templates", International Journal of Computer Science and Network, Vol 5, No 4, pp 615 - 621, 2016.

9. Kandhasamy, J.P., Balamurali, S., Kadry, S, R. Lakshmana Kumar,' Diagnosis of diabetic retinopathy using multi level set segmentation algorithm with feature extraction using SVM with selective features," Multimedia Tools Applications https://doi.org/10.1007/s11042-019-7485-8

10. C. Aravind, M. PonniBala, S. Vijayachitra ,"Automatic detection of microaneurysms and classification of diabetic retinopathy images using SVM technique", International Journal of Computer Applications- International Conference on Innovations In Intelligent Instrumentation, Optimization And Signal Processing “ICIIIOSP-2013”,pp. 18-22.

11. Kandhasamy JP and Balamurali S "Performance analysis of classifier models to predict diabetes mellitus". Procedia Computer Science, Vol. 47, pp. 45-51. 2015

12. T. Kauppi, V. Kalesnykiene, Joni-K. Kamarainen, L. Lensu, I. Sorri A. $\underline{\text { Raninen, R. Voutilainen, H. Uusitalo, H. Kälviäinen, J. Pietilä }}$ "DIARETDB1 diabetic retinopathy database and evaluation protocol", Proceedings of the British Machine Vision Conference 2007, pp. 1-18.

13. A. Rocha, T. carvalho, Herbert F. Jelinek, S, Goldensten, J. Wainer, "Points of interest and visual dictionaries for automatic retinal lesion detection," IEEE Transaction on Biomedical Engineering, Vol. 59, No. 8, pp. 2244-2253, 2012.

14. S. S. Rahim, V. Palade, C. Jayne, A. Holzinger, J. Shuttleworth, "Detection of diabetic retinopathy and maculopathy in eye fundus images using Fuzzy image processing", lecture notes in computer science, pp 379-388, 2015

\section{AUTHORS PROFILE}

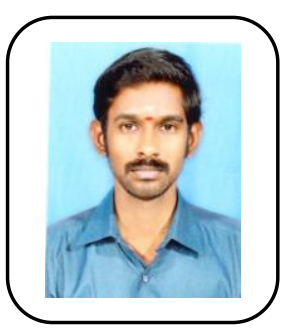

Mr. J. Pradeepkandhasamy, Assistant Professor, Department of Computer Applications, Kalasalingam Academy of Research and Education have more than 7 years of Teaching Experience. He completed his Under Graduation in Computer Science at 
Bharathidasan University and Post Graduation in Master of Computer Applications in Kalasalingam University, His Major research area is prediction and classification in Data Mining

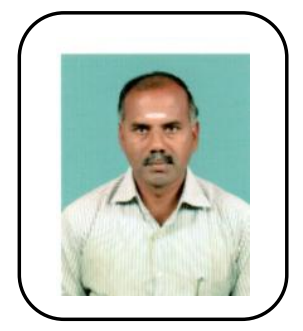

Dr. S. Balamurali, Senior Professor, Department of Computer Applications, Kalasalingam Academy of Research and Education, he has more than 30 years of Teaching Experience, he has completed his Under graduate in Mathematics in PSG College of Arts and Science, Post Graduate in Statistics in PSG in College of Arts and Science and Doctoral Degree from Bharathiar University in the field of Statistics, His current area of Research is Statistics, Data Mining, Network Security. He has more than 200 publications in International Journals. He is acting as a reviewer for more than 20 peer reviewed journals.

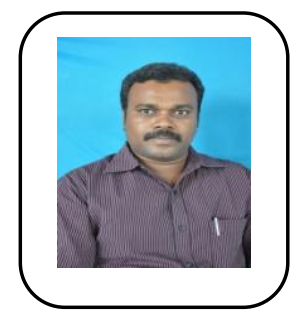

Mr. M. Arun, Assistant Professor, Department of Computer Applications, Kalasalingam Academy of Research and Education have more than 12 years of Teaching Experience. He completed his Under Graduation in Computer Applications at Madurai Kamarajar University and Post Garduation in Computer Applications at Anna University, His Major research area is Network Security.

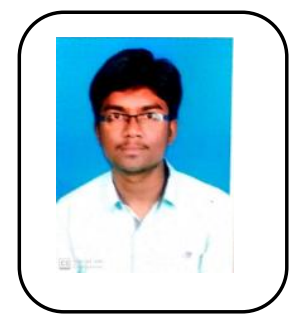

M. Mariappan is a student doing his masters in Computer Applications at Kalasalingam Academy of Research and Education; he had done his Bachelor's degree in Computer Applications at same university. His research area is data mining and image processing. He is well versed in Designing poster, photos. 